MPP-2012-121

\title{
Investigating Gamma-Ray Lines from Dark Matter with Future Observatories
}

\author{
Lars Bergström团 Jan Conrad \\ The Oskar Klein Centre for Cosmoparticle Physics, Department of Physics, \\ Stockholm University, AlbaNova, SE-106 91 Stockholm, Sweden
}

Gianfranco Berton£§

GRAPPA Institute, University of Amsterdam, Science Park 904, 1090 GL Amsterdam, Netherlands

\author{
Christoph Weniger \\ Max-Planck-Institut für Physik, Föhringer Ring 6, 80805 München, Germany
}

\begin{abstract}
We study the prospects for studying line features in gamma-ray spectra with upcoming gammaray experiments, such as HESS-II, the Cherenkov Telescope Array (CTA), and the GAMMA-400 satellite. As an example we use the narrow feature at $130 \mathrm{GeV}$ seen in public data from the FermiLAT satellite. We found that all three experiments should be able to confidently confirm or rule out the presence of this $130 \mathrm{GeV}$ feature. If it is real, it should be confirmed with a confidence level higher than $5 \sigma$. Assuming it to be a spectral signature of dark matter origin, GAMMA-400, thanks to a projected energy resolution of about $1.5 \%$ at $100 \mathrm{GeV}$, should also be able to resolve both the $\gamma \gamma$ line and a corresponding $Z \gamma$ or $H \gamma$ feature, if the corresponding branching ratio is comparable to that into two photons. It will also allow to distinguish between a gamma-ray line and the similar feature resulting from internal bremsstrahlung photons.
\end{abstract}

PACS numbers:

\section{INTRODUCTION}

As the Large Hadron Collider (LHC) keeps accumulating data at high luminosity (and soon at full energy), hopes are high that it will help elucidating the nature of the particle making up around $23 \%$ of the energy density of the universe, the dark matter particle [1, 2]. So far, no such new mass scale has been found, although the prediction from supersymmetric (SUSY) models that the lightest Higgs boson should weigh less than $130 \mathrm{GeV}[3]$, which seems to be confirmed by the detection recently done at CERN's LHC, which gives a mass of the potential Higgs boson of around $125 \mathrm{GeV}$.

As for dark matter candidates, only constraints on the parameter space of the most popular extensions of the Standard Model, in particular Supersymmetry, have been obtained [4], but even if such candidates were to be found, it will be hard to prove with LHC data only that they actually constitute most of the dark matter in the Universe, as the required lifetime of many times the age of the universe would seem impossible to verify in accelerator experiments [5].

Fortunately, direct and indirect dark matter searches will provide complementary information, possibly allowing a precise identification of dark matter particles [6, 7]. Direct detection by scattering of dark matter particles

\footnotetext{
*Electronic address: lbe@fysik.su.se

${ }^{\dagger}$ Electronic address: conrad@fysik.su.se

¥Electronic address: Christian.Farnier@fysik.su.se

$\S$ Electronic address: gf.bertone@gmail.com

ฯElectronic address: weniger@mpp.mpg.de
}

traversing the earth in ultra-pure counting experiments has historically been the most advanced technique, but indirect detection methods have recently received increased interest (see e.g. Ref. [1] for reviews).

Indirect detection is based on the search for secondary photons, antimatter, and neutrinos produced by the annihilation or decay of dark matter particles. For $\gamma$-rays coming from annihilations of dark matter particles in the halo, Fermi-LAT has very successfully delivered bounds that have started to probe into the parameter space of viable models, in line with pre-launch expectations [8], in particular for dwarf spheroidal galaxies [9] and galaxy clusters [10].

Recently, a possible hint of a dark matter signal in the form of a narrow spectral line or an internal bremsstrahlung (IB) feature, has been found in analyses of public data from the Fermi-LAT satellite detector [11, 12] (see also [13, 14]). The signal is too weak to claim a discovery, but being of a type and at an energy where there is no other known astrophysical explanation ${ }^{1}$ it is important to further study this type of signature in independent experiments.

We take in this paper, as an exercise, the existence of these recent indications for a line or an IB bump seriously, and we discuss how this effect, if real, would appear in a number of existing (Fermi-LAT 16], HESS-II [17]) and planned (CTA [18], GAMMA-400 [19]) $\gamma$-ray detectors. If the present indications of a line structure in the FermiLAT public data would disappear, our results should be

\footnotetext{
${ }^{1}$ The very fine-tuned pulsar model from [15] can be disregarded since the signal is significantly extended.
} 
useful for future indirect dark matter searches.

In particular, we discuss the possibility of one or more associated lines coming from the $Z \gamma$ and $H \gamma$ (with $H$ the Higgs boson) annihilation channels in some models, and we investigate whether one can separate a line signal from other spectral features like IB emission. We will show that with the upcoming detector HESS-II, and the proposed Cherenkov Telescope Array (CTA) and the GAMMA-400 satellite these gamma-ray structures, if real, indeed will be confirmed with much higher confidence.

The paper is organized as follows: in Sec. II we review the spectral features arising from dark matter annihilation and the tentative detection of a feature in Fermi data. In Section [II] we discuss the prospects to detect such feature with future observatories focusing on improvements in energy resolution and effective area. In IV we discuss our results and present our conclusions.

\section{INDIRECT DETECTION WITH GAMMA-RAYS}

\section{A. Basics}

Among the possible secondary particles, gamma-rays play a special role in indirect dark matter searches, since they propagate without being absorbed or deflected in the local Universe, in contrast, e.g., to the case of antimatter (see [2]).

The expected dark matter generated gamma-ray flux from a cone with solid angle $\Delta \Omega$ observed at earth can be written

$$
\frac{d \Phi_{\gamma}}{d E}=\frac{\langle\sigma v\rangle}{2 m_{\chi}^{2}} \sum_{f} \frac{d N_{\gamma}^{f}}{d E} \times \frac{B}{4 \pi} \int_{\Delta \Omega} d \Omega \int_{\text {l.o.s. }} d \lambda \rho_{\mathrm{dm}}^{2}(\lambda),
$$

where $\langle\sigma v\rangle$ is the total average annihilation rate, $N_{\gamma}^{f}$ the number of photons produced in annihilation channel $f$, $\rho_{\mathrm{dm}}$ the smooth part of the dark matter density and $\lambda$ the line-of-sight distance in the direction of observation. This expression has to be convolved with the energy and angular resolution of the detector.

The dark matter density along the line of sight, $\rho_{\mathrm{dm}}(\lambda)$, is at present unconstrained in the inner Galaxy, and it is therefore derived from N-body simulations, and most commonly approximated with analytic fits like the NFW [20] or the Einasto profile [21], for which the integral along the line of sight turns out to produce similar values. In the rest of the paper we make use of the Einasto profile,

$$
\rho_{\mathrm{dm}}(r) \propto \exp \left(-\frac{2}{\alpha_{E}} \frac{r^{\alpha_{E}}}{r_{s}^{\alpha_{E}}}\right),
$$

with $\alpha_{E}=0.17$ and $r_{s}=20 \mathrm{kpc}$, normalized to $\rho_{\mathrm{dm}}\left(r_{0}\right)=0.4 \mathrm{GeV} \mathrm{cm}^{-3}$ at position of the Sun, $r_{0}=$ $8.5 \mathrm{kpc}$.

A boost factor $B$ is often included in the calculation, to parametrize our ignorance on the enhancements of the annihilation signal that may be caused by processes like: the presence of dark matter substructures; the so-called Sommerfeld enhancement factor 22] (although the possibility of a very large $B$ factor is constrained by Cosmic Microwave Background observations [23]) or strong deviations of the dark matter distribution from the Einasto or NFW profiles.

As it has been clear for quite some time, it is not possible to reliably predict the size of the boost factor $B$, as it (besides the particle physics uncertainties relating to the Sommerfeld enhancement) depends critically on the density profile and spatial distribution of subhalos, a problem largely beyond the capability of present-day simulations. What we know is that numerical simulations suggest that there should be fewer substructures in the inner galaxy than in the outer parts, due to the process of merger and tidal stripping in the Milky Way halo (see e.g. 24, 25] and references therein), and that baryons dominate the gravitational potential at the centre of the Galaxy, where galactic baryons such as the bar and the central supermassive black hole strongly modify the distribution of dark matter [26].

The dependence of the annihilation rate on the square of the dark matter density may cause "hot spots" near density concentrations such as dwarf galaxies, although recently it has appeared that galaxy clusters may be even more interesting targets for detection claims [27]. However, the strongest signal should exist near the Galactic centre, as predicted by all halo modeling, including Nbody simulations, and we will concentrate on that target throughout this work.

\section{B. Identity of a $\gamma$-ray line signal}

Especially important for the clear demonstration of a dark matter signal is the possibility of "smoking gun" signatures like gamma-ray lines [28, 29] or similar structures caused by internal bremsstrahlung (IB) [30]. Presently no known astrophysical background processes that can give such conspicuous signals have been observed.

Depending on the structure of the theory of the dark matter particle $\chi$, one can imagine at present at least three different origins of a sizeable narrow line. The most obvious (and in many cases the strongest) will come from the $\chi \chi \rightarrow \gamma \gamma$ process, which will give a line at energy $E_{\gamma}=m_{\chi}$, with a very narrow intrinsic width that will be dominated by Doppler motion of the annihilating pair, $\epsilon_{\gamma} \equiv \delta E_{\gamma} / E_{\gamma} \simeq 10^{-3}$ for typical Galactic velocities. For annihilation into another neutral particle plus a photon, like the electroweak gauge boson $Z^{0}$ or the Higgs boson $H$, the line width will be dominated by the intrinsic width of the decay particle, which for the $Z^{0}$ case will give $\epsilon_{\gamma} \simeq 10^{-2}$, and smaller for a standard model Higgs boson, if it coincides with the detection claimed at LHC. The relation between the photon energy and the mass of the 


\section{$\chi \chi$ to $\mathrm{P} \gamma$ for $\mathrm{P}=\gamma, \mathrm{Z}, \mathrm{H}$}

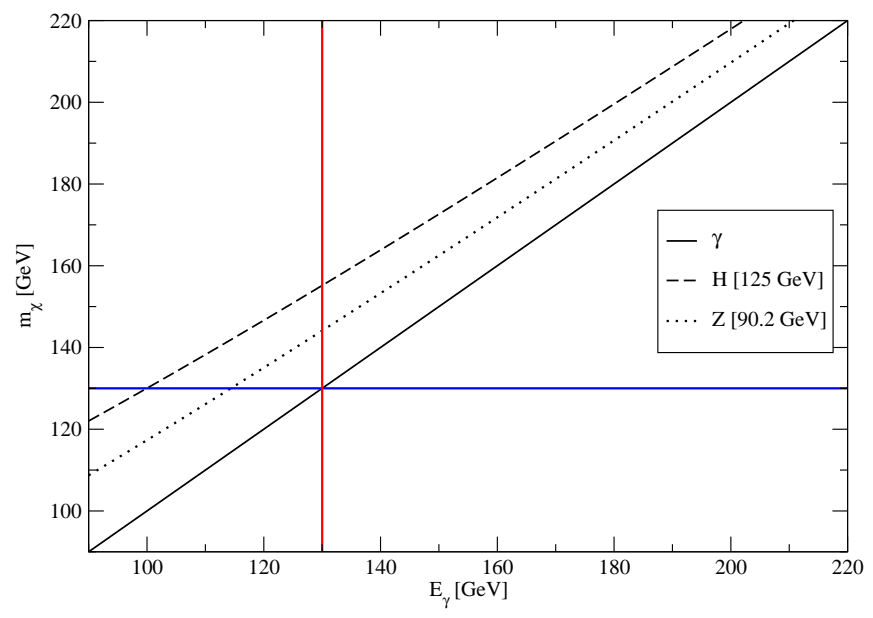

FIG. 1: Illustration of Eq. (4), which relates the mass $m_{\chi}$ of the annihilating particles with the energy of the $\gamma$-ray line,for the case $\chi \chi \rightarrow P \gamma$, with $P=\gamma, H, Z^{0}$. Here $m_{Z}$ is $90.2 \mathrm{GeV}$, and as an illustration the Higgs mass has been set to $125 \mathrm{GeV}$, indicated by present LHC data [3].

particle $P$ in the final state is

$$
E_{\gamma}=m_{\chi}\left(1-\frac{m_{P}^{2}}{4 m_{\chi}^{2}}\right)
$$

or, solving instead for $m_{\chi}$

$$
m_{\chi}=\frac{1}{2}\left(E_{\gamma}+\sqrt{m_{P}^{2}+E_{\gamma}^{2}}\right) .
$$

This relation is shown in Fig. 1. Assuming that the observed gamma-ray line at $130 \mathrm{GeV}$ is due to the $\chi \chi \rightarrow \gamma \gamma$ process one finds, following the horizontal line, predictions for the location of $H \gamma$ and $Z^{0} \gamma$ lines at $100 \mathrm{GeV}$ and $114 \mathrm{GeV}$, respectively. Alternatively, following the vertical line, one sees that if the observed $130 \mathrm{GeV}$ line is a result of the $\chi \chi \rightarrow H \gamma$ or $Z^{0} \gamma$ process, the $\chi$ mass is 155 or $142 \mathrm{GeV}$, respectively.

\begin{tabular}{|l|l|l|l|}
\hline & $\gamma \gamma$ & $H \gamma$ & $Z \gamma$ \\
\hline$\gamma \gamma$ & 130 & 100 & 114 \\
\hline$H \gamma$ & 155 & 130 & 142 \\
\hline$Z \gamma$ & 144 & 117 & 130 \\
\hline
\end{tabular}

TABLE I: Predicted $\gamma$-ray energies, in $\mathrm{GeV}$, if the $130 \mathrm{GeV}$ line originates from the process indicated by the row, for the process given by the respective column.

The predicted energies of all three possible lines, the ones coming from $\gamma \gamma, H \gamma$ and $Z^{0} \gamma$ (with $m_{H}$ set to 125
$\mathrm{GeV}$ ), for all permutations are shown in Table \ It will depend on the model if all three lines are allowed. In particular, as a radiative $0 \rightarrow 0$ transition is forbidden due to gauge invariance and angular momentum conservation, the annihilation to $H \gamma$ is not allowed from the dominant $s$ wave in the Galaxy if $\chi$ is a Majorana fermion or a spin-0 particle [31]. For definiteness, we will in the following assume that the tentative $130 \mathrm{GeV}$ structure is due to the $\gamma \gamma$ line, but we will also compare with the expectations for the IB effect, to which we now turn.

\section{The internal bremsstrahlung effect}

The $\gamma \gamma$ process normally appears in a closed loop containing the various charged particles to which the dark matter particles couple. This means that it is generally suppressed by powers of the electromagnetic coupling constant, and the cross section will contain an explicit factor $\alpha_{e m}^{2}$. An interesting effect appears, however, for Majorana fermions already at order $\alpha_{e m}$. It was early realized that there could be important spectral features [32], and recently it has been shown that internal bremsstrahlung (IB) from charged particles in the $t$-channel in the annihilations could yield a detectable, quite sharp "bump" near the highest energy, i.e., at the rest mass of one of the annihilating particles moving slowly $\left(v / c \sim 10^{-3}\right)$ in the Galactic halo [11, 30, 33]. In [34], it was furthermore pointed out that final state radiation (FSR) often can be estimated by simple, universal formulas and often gives rise to a very prominent step in the spectrum at photon energies of $E_{\gamma}=m_{\chi}$. The IB and FSR processes was thoroughly treated in [33] (see also $[11,30 \mid$ ), and here we summarize the main results.

In Ref. [32] it was shown that the radiative process $\chi^{0} \chi^{0} \rightarrow f \bar{f} \gamma$ may circumvent the chiral suppression, i.e., the annihilation rate being proportional to $m_{f}^{2}$. This is normally what one would get for annihilation into a fermion pair from an $s$-wave initial state [35], as is the case in lowest order for non-relativistic dark matter Majorana particles in the Galactic halo. A fermion final state with an additional photon, $f \bar{f} \gamma$, is thus surprisingly not subject to a helicity suppression. The full analytical expressions are lengthy, but simplify in the limit of $m_{f} \rightarrow 0$. Then one finds in the supersymmetric case 33. for the radiative differential rate, normalized to the $f \bar{f}$ rate

$$
\frac{d N_{f}^{\gamma, \mathrm{IB}}}{d x}=
$$

$\Delta \times\left[\frac{4 x}{\mu(\mu-2 x)}-\frac{2 x}{(\mu-x)^{2}}-\frac{\mu(\mu-2 x)}{(\mu-x)^{3}} \log \frac{\mu}{\mu-2 x}\right]$,

with

$$
\Delta=(1-x) \alpha_{\mathrm{em}} Q_{f}^{2} \frac{\left|\tilde{g}_{R}\right|^{4}+\left|\tilde{g}_{L}\right|^{4}}{64 \pi^{2}}\left[m_{\chi}^{2}\langle\sigma v\rangle_{\chi \chi \rightarrow f \bar{f}}\right]^{-1},
$$


where $Q_{f}$ is the electric charge of the fermion, $\mu \equiv$ $m_{\tilde{f}_{L, R}}^{2} / m_{\chi}^{2}+1$ and $\tilde{g}_{R} P_{L}\left(\tilde{g}_{L} P_{R}\right)$ is the coupling between the dark matter particle, the fermion and right-handed (left-handed) $t$-channel exchange particle $\tilde{f}$ (which would be a sfermion in the supersymmetric case). This agrees with the result found in 32] for the case of pure photino annihilation (see [36] for a right-handed neutrino dark matter candidate where this interesting internal bremsstrahlung effect also appears). Note the large enhancement factor $m_{\chi}^{2} / m_{f}^{2}$ due to the lifted helicity suppression (from $\langle\sigma v\rangle_{\chi \chi \rightarrow f \bar{f}} \propto m_{f}^{2} m_{\chi}^{-4}$ ), and another large enhancement that appears at high photon energies for sfermions degenerate with the neutralino.

For the supersymmetric case, internal bremsstrahlung from the various possible final states of neutralino annihilations is included in DarkSUSY [37]. The total $\gamma$-ray spectrum is given by

$$
\frac{d N^{\gamma, \text { tot }}}{d x}=\sum_{f} B_{f}\left(\frac{d N_{f}^{\gamma, \text { sec }}}{d x}+\frac{d N_{f}^{\gamma, \text { IB }}}{d x}+\frac{d N_{f}^{\gamma, \text { line }}}{d x}\right),
$$

where $B_{f}$ denotes the branching ratio into the annihilation channel $f$. The last term in the above equation gives the contribution from the direct annihilation into photons, $\gamma \gamma$ or $Z \gamma$, which as mentioned before result in a sharp line feature [28, 29]. The first term is the contribution from secondary photons from the fragmentation of the fermion pair. This "standard" part of the total $\gamma$-ray yield from dark matter annihilations shows a feature-less spectrum with a rather soft cutoff at $E_{\gamma}=m_{\chi}$.

In Fig. 2 an example of the energy distribution of photons given by the first two terms in (6) is shown. To this may be added one or more line signals, the strength of which is very model dependent, however.

\section{Brief review of present indications of $\gamma$-ray line signal}

Past searches for gamma-ray lines in the Fermi-LAT or EGRET data found no indications for line signatures below $200 \mathrm{GeV}$, and presented upper limits on the annihilation cross-section (or decay rate, in some models) of dark matter particles into monochromatic photons 38 42.

However, recent analyses of public Fermi-LAT data [16] have identified a feature at $130 \mathrm{GeV}$ in a line-search from the halo in the vicinity of the galactic centre, that has tentatively been interpreted in terms of dark matter [11, 12]. Beside taking into account the full set of available data, the major improvement with respect to previous studies was an adaptive selection of search regions with optimized signal-to-noise level for different profiles of the Galactic dark matter halo.

The revealed signature is too sharp to allow a straightforward astrophysical explanation, and strongly suggestive towards an interpretation in terms of a gamma-ray
Internal bremsstrahlung spectrum

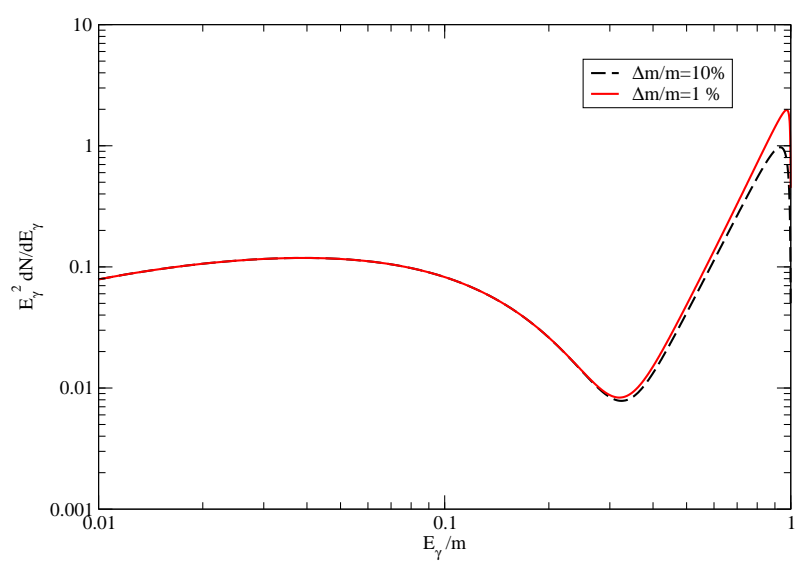

FIG. 2: The distribution of $\gamma$-rays from the internal bremsstrahlung process $\chi \chi \rightarrow f \bar{f} \gamma$ (see [11]), for two different values of the relative mass difference between the $t$-exchange particle and the dark matter Majorana fermion $\chi$. (Here a continuous spectrum of the $b \bar{b}$ type is also included.) As can be seen, the internal bremsstrahlung process gives a very hard spectrum which peaks near the kinematical end point, and thus is a "smoking gun" signature for dark matter annihilation, similar to the $\gamma$-ray line signal.

line at $130 \mathrm{GeV}$ [12], or alternatively as the virtual internal bremsstrahlung from dark matter particles with a mass of around $150 \mathrm{GeV}$ [1]. The signature appears close to the Galactic centre only.

This discovery has already gained considerable attention (see, e.g., [43]). Ref. [44] suggested the Fermi Lobes (bubbles/haze) [45] as cause for the observed signature; but this possibility was however quickly disputed by Ref. [13] and Ref. [14], who emphasized the locality of the signature close to the Galactic centre and relaxed the power-law approximation to the background fluxes.

In particular, in the analysis in [14] a signal of more than $5 \sigma$ was found, but caveats of the interpretation are also given: there is a modest evidence of an $1.5^{\circ}$ offset of the signal region with respect to the Galactic centre (this may however be explained by the interplay between baryons and dark matter [46]), and there are weak indications of a possible instrumental effect giving spurious lines of which one also happens to be at $130 \mathrm{GeV}$. In either case, whether these new results are a consequence of a genuine dark matter signal, or point to an unexpected instrumental effect of the Fermi-LAT detector, it is imperative that this signal region is probed by independent detectors.

If interpreted in terms of dark matter annihilation, the signature is consistent with an Einasto or NFW profile [12, 14]. In this case, the annihilation cross-section for $\chi \chi \rightarrow \gamma \gamma$ is best given by $\langle\sigma v\rangle \simeq 1.3 \cdot 10^{-27} \mathrm{~cm}^{3} \mathrm{~s}^{-1}$, with a dark matter mass of $m_{\chi} \simeq 129.8 \pm 2.4_{-13}^{+7} \mathrm{GeV}$ [12], or $127.0 \pm 2.0 \mathrm{GeV}$ [14]. 


\section{PROSPECTS FOR IDENTIFYING SPECTRAL FEATURES WITH FUTURE OBSERVATORIES}

\section{A. Statistical treatment}

In this work, we study the prospects for detecting and identifying sharp gamma-ray signatures (possibly arising from dark matter annihilation) with GAMMA-400, HESS-II and CTA, by adopting the standard methods that were used in previous line searches [12, 39 42] and apply them to different sets of appropriately generated mock data. ${ }^{2}$ For each instrument under consideration, the basic strategy is: (1) Determine a region of the sky with large expected signal-to-noise ratio; this is typically the Galactic centre, maybe plus regions above/below the Galactic disc. (2) Perform a spectral analysis of the (here simulated) gamma-ray fluxes in this region using the maximum likelihood technique [47].

Throughout, best-fit model parameters are inferred by maximizing the likelihood function $\mathcal{L}=\Pi_{i} P\left(c_{i} \mid \mu_{i}\right)$, where $P(c \mid \mu)$ is the Poisson probability to observe $c$ events when $\mu$ are expected, $c_{i}$ denotes the number of events that fall into energy bin $i$, and $\mu_{i}$ is the number of expected events in bin $i$ and a function of the model parameters and the instrument response function. All fits are only performed within small energy windows centred around the line position(s). The small energy windows allow to approximate astrophysical background fluxes locally by a single power-law (we leave normalization and spectral slope free in the fits), and it eliminates the need to include secondary signal photons into the fits (because they would peak far outside the window). The energy window ranges per definition from $E_{\gamma} / \sqrt{\epsilon}$ to $E_{\gamma} \sqrt{\epsilon} ; E_{\gamma}$ is the line energy, the energy window size $\epsilon$ is typically a factor of a few larger than the energy resolution of the respective instrument and will be defined below. Note that in all fits we keep the position of the line-like features fixed. Our treatment of the instrument response functions that we need in order to obtain $\mu_{i}$ are described below; fits are performed using the ISIS package [48].

Projected upper limits at the $95 \% \mathrm{CL}$ are derived using the profile likelihood method [49]: We generate a large number of mock data sets without a signal. We then fit the mock data with a power-law with free slope and normalization plus a line signal with free non-negative normalisation and increase the line flux from its best-fit value until $-2 \ln \mathcal{L}$ changed by 2.71 (while profiling over the background parameters). For different line energies, we quote the log-scale mean of the limits obtained this way as the expected limit.

The local significance for a line signature can be derived using the maximum-likelihood-ratio test; it

\footnotetext{
2 A complementary approach that could be adopted in the analysis of GAMMA-400 data would be a spatial analysis as in Ref. [14].
}

is given by the square-root of the TS-value $T S \equiv$ $-2 \ln \left(\mathcal{L}_{\text {null }} / \mathcal{L}_{\text {alt }}\right)$, where $\mathcal{L}_{\text {null }}$ and $\mathcal{L}_{\text {alt }}$ are the likelihoods of background-only (a single power-law) and backgroundplus-line fits, respectively. For the different experiments, we determine the $5 \sigma$ detection threshold for monochromatic photons by adjusting the signal flux such that the TS-value averaged over a large number of mock data sets is 23.7 [50].

In case a narrow line is identified, it is imperative to devise a strategy to discriminate between IB and monochromatic photons, and to test for the presence of additional closeby lines. We are interested in the flux at which such a discrimination becomes possible at the 95\% CL. To this end, we simulate data with IB features or with two lines (corresponding to $\gamma \gamma$ and $\gamma P$ final states, with $P=\mathrm{H}, \mathrm{Z})$, and perform a maximal likelihood ratio test to compare the likelihood of $\mathrm{IB} /$ twoline fits with the one-line scenario. We search for the flux for which the average of the log-likelihood ratio is $\left\langle-2 \ln \left(\mathcal{L}_{\text {one-line }} / \mathcal{L}_{\text {two-lines } / \text { VIB }}\right)\right\rangle=4$. In case of the twoline model, we leave the relative normalization of the two lines, as well as their position, fixed. The IB signal from dark matter with mass $m_{\mathrm{dm}}$ is compared with a monochromatic line at $E_{\gamma} \approx 0.90 \mathrm{~m}_{\mathrm{dm}}$, since the IB feature peaks slightly below the kinematical threshold; in these fits the dark matter mass is left as a free parameter. Since the models are not nested, the interpretation of the log-likelihood ratio is in principle not straightforward. However, the obtained significance levels are conservative with respect to what is obtained in a comprehensive likelihood test [51]; for a study of the comprehensive likelihood method in the context of dark matter searches see [52].

\section{B. Effects of energy resolution - The case of GAMMA-400}

The search of a weak gamma-ray line-like signal on top of a continuous background spectrum may be hindered by statistical fluctuations of the background. This is particularly true if the signal is additionally spread over a large energy range due to the limited energy resolution of the instrument. For pair conversion gamma-ray space telescopes, one of the main instrumental characteristics influencing the resolution at high energies is the calorimeter dimensions, measured in radiation lengths $\left(X_{0}\right)$. This is because the energy reconstruction at high energies is crucially depending on how well the electromagnetic shower can be mapped. Fermi-LAT with its 8 radiation lengths reaches an energy resolution of around $\sim 10 \%$ at energies of about $100 \mathrm{GeV}$.

The design of the GAMMA-400 experiment, a future Russian-Italian pair conversion telescope whose preliminary launch date is announced to 2018 [19], should be similar to that of the Fermi-LAT. It will consist of a tracker unit, a calorimeter and anti-coincidence systems. Design details, such as number of tracker layers, presence 


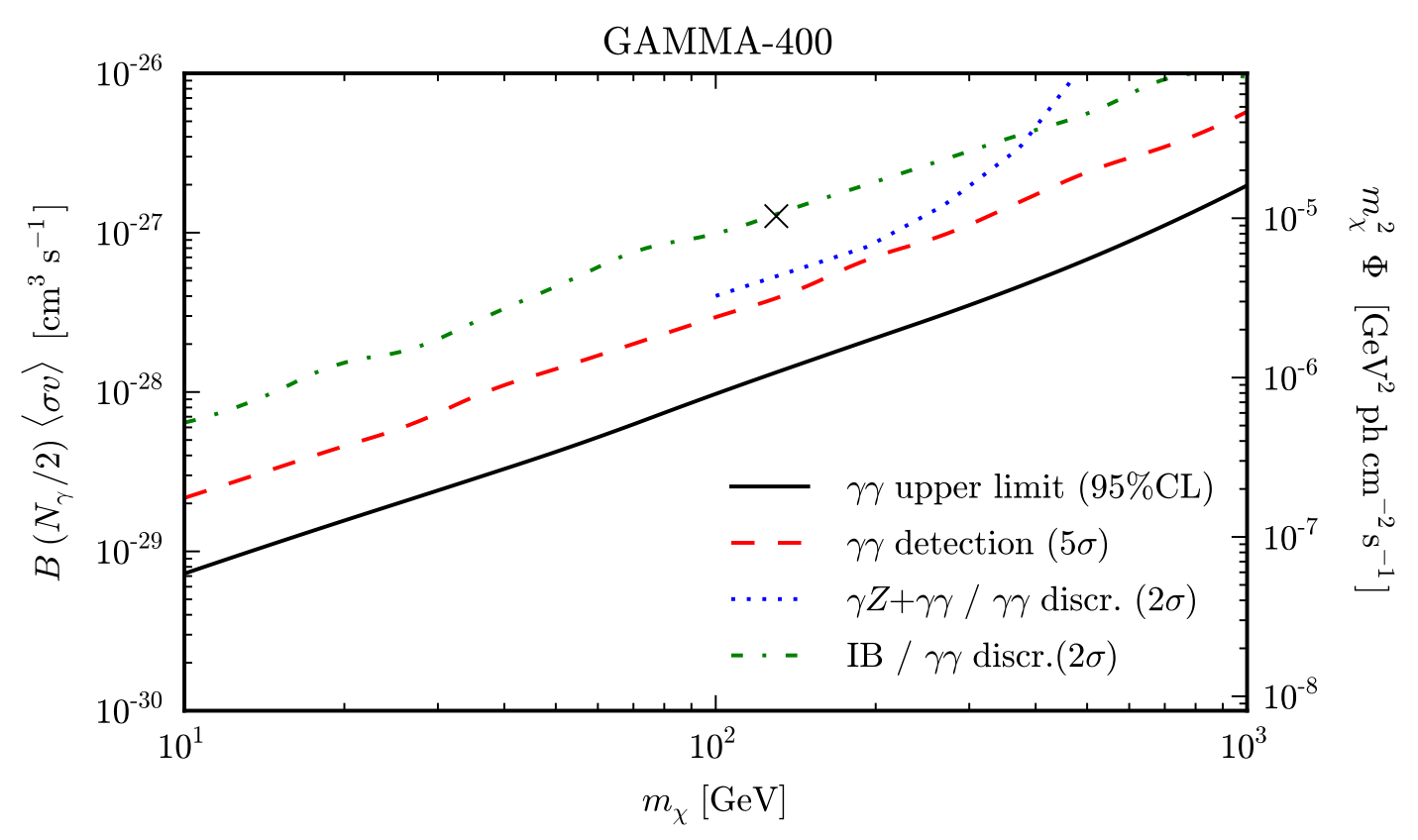

FIG. 3: Estimated flux and velocity weighted cross-section sensitivities obtained for GAMMA-400 as a function of the DM particle mass. Black solid: Expected upper limits for $\gamma \gamma$ final states (with $N_{\gamma}=2$ ); red dashed: detection of $\gamma \gamma$ at $5 \sigma$; green dot-dashed: discrimination between IB and a monochromatic line $\left(N_{\gamma}=1\right)$; blue dotted: discrimination between $\gamma Z+\gamma \gamma$ and $\gamma \gamma$ (assuming $\left.\operatorname{BR}(\chi \chi \rightarrow \gamma \gamma)=\mathrm{BR}(\chi \chi \rightarrow \gamma Z)=0.5 ; N_{\gamma}=1.5\right)$. The cross indicates the $130 \mathrm{GeV}$ signature from Ref. [12].

of converter material in the tracker and design of anticoincidence systems (which will be very demanding due to a very excentric orbit) are not fixed yet, but it is very probable that GAMMA-400 will be equipped with a 22 $X_{0}$ calorimeter with claimed energy resolution of about $1.5 \%$ at $100 \mathrm{GeV}$.

The effective area of GAMMA-400 is however foreseen to be smaller (around $6400 \mathrm{~cm}^{2}$ ) above a few tens of $\mathrm{GeV}$ and constant. These two design features immediately allow a rough estimate of the expected improvement: for upper limits $\sqrt{2 / 10} \sim 0.4$, for signal significance $10 / 2 \sim 5$. Interestingly, a larger calorimeter also will help with background rejection (which is largely based on shower shape) and extend the usable energy range to larger energies. As mentioned, the design phase is not concluded and the design still allows considerable freedom. Here, we assume the energy resolution of GAMMA400 to scale as the one of the Fermi-LAT (such that it ranges from $3 \%$ at $10 \mathrm{GeV}$ to $1 \%$ at $1 \mathrm{TeV}$ ).

With the hypothesis that the final design of GAMMA400 is similar to the one described above, we derived the sensitivity of GAMMA-400 for spectral signatures from dark matter annihilation after 5 years of full sky survey mode operation. As target region, we select a circular region around the Galactic centre with $20^{\circ}$ radius excluding the Galactic disc part $\left(|\ell|>5^{\circ} \&|b|<5^{\circ}\right)$. To model the background that will be observed by GAMMA-400, we make use of the current observations of the gammaray emission observed by the Fermi-LAT as reported in 12]; the events observed above few $\mathrm{GeV}$ in our region of interest can be described by a power-law function $\mathrm{dN} / \mathrm{dE}_{\mathrm{bckg}}=6 \cdot 10^{-11} \times\left(\frac{E}{1 \mathrm{GeV}}\right)^{-2.5} \mathrm{ph} \mathrm{GeV}^{-1} \mathrm{~cm}^{-2} \mathrm{~s}^{-1}$. The exposure time of the source region is $3.2 \times 10^{7} \mathrm{~s}$, assuming a field-of-view of $2.4 \mathrm{sr}$. In our spectral fits, we will adopt the energy windows from Ref. 12], ranging from $\epsilon=1.5$ at $10 \mathrm{GeV}$ to $\epsilon=3.1$ at $1 \mathrm{TeV}$. We checked that our results do not critically depend on this choice.

In Fig. 3, our results for the projected 95\%CL upper limits from GAMMA-400 are shown by the black solid line. Even if the effective area is smaller than the one of Fermi-LAT, the better energy resolution of the instrument allows to better distinguish a deviation due to a line signal from the power law background fluctuations. This would improve by a factor about two to three the upper limits that are obtained by Fermi-LAT in the same observational time.

We also computed the strength of a line-like signal in order to obtained a $5 \sigma$ level detection for GAMMA400 after 5 years of survey mode. In this case, the $\gamma \gamma$ annihilation cross-sections range between $10^{-29}$ and 5 . $10^{-27} \mathrm{~cm}^{3} \mathrm{~s}^{-1}$, depending on the mass of the DM particle (see red dotted line on Fig. 3). The confirmation of the tentative $130 \mathrm{GeV}$ line at $5 \sigma$ would require $\sim 20$ months in survey mode, and $\sim 10$ months using pointed observation.

For such good energy resolution performances, the instrument should also be able to probe efficiently the existence of a secondary line. As an illustrative example, 


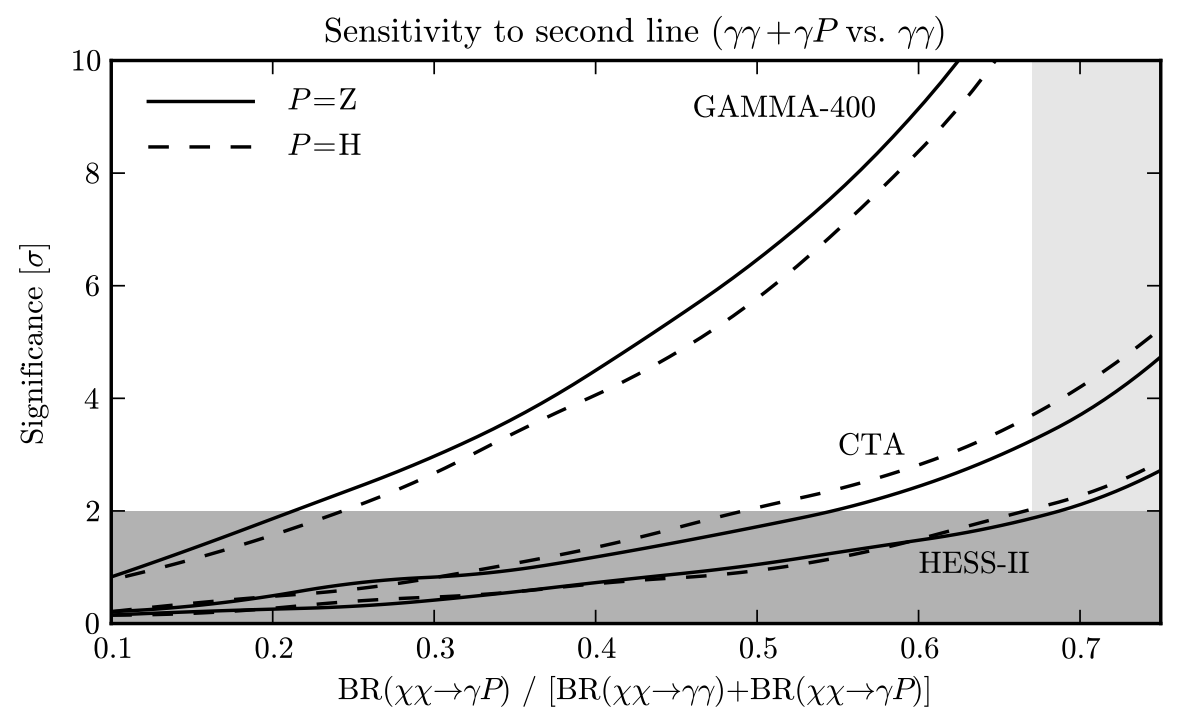

FIG. 4: Assuming that the $130 \mathrm{GeV}$ feature from Ref. [12] is due to annihilation into $\gamma \gamma$, we show as function of the branching ratios the significance at which a second line (either $\gamma \mathrm{Z}$ or $\gamma \mathrm{H}$ ) could be detected by different experiments. In the right gray area the secondary line would be as strong as the primary one; in the lower gray area the significance would fall below $2 \sigma$.

we consider here the case that dark matter annihilates into $\gamma \gamma$ and $\gamma Z$ final states with the same rate. For such scenarios, GAMMA-400 will be able to distinguish a twoline model from a single line one at 95\% CL in the region indicated by the blue dotted line in Fig. 3. At energies above few hundred $\mathrm{GeV}$, both lines start to overlap, and a distinction becomes impossible.

Assuming that the $130 \mathrm{GeV}$ feature from Ref. [12] is produced by annihilation into $\gamma \gamma$ final states, we calculated the significance at which GAMMA-400 will be able to identify secondary lines from either $\gamma \mathrm{Z}$ or $\gamma \mathrm{H}$ (see Tab. I) for different branching ratios. In Fig. (4) we show the expected significance as function of the branching ratio into the secondary line. As one can see from this plot, GAMMA-400 would be an important probe for additional lines, down to $\operatorname{BR}(\chi \chi \rightarrow \gamma P) /$ $\operatorname{BR}(\chi \chi \rightarrow \gamma \gamma) \sim 0.2$.

Finally, we investigate for which signal fluxes a signal arising from the annihilation into $\gamma \gamma$ can be distinguished from IB photons. We fix here the mass splitting (see above) to $\mu=2.1$ for definiteness. In Fig. 3. we show by the green dash-dotted line the annihilation cross-sections for which GAMMA-400 would be able to distinguish at a 95\% CL between monochromatic photons and IB photons. In particular, a study of the nature of the $130 \mathrm{GeV}$ excess seems realistic.

\section{Effects of increased area - The case of CTA and HESS-II}

Space-based experiments are generally strongly size limited. The Fermi-LAT is currently the largest pair- conversion telescope ever launched, and its effective area is below $1 \mathrm{~m}^{2}$. This particularly reduces the possibility of gamma-ray astronomy in the $\mathrm{TeV}$ domain, since the number of observed events would be less than one per year even for the strongest steady source. $\mathrm{TeV}$ astronomy is therefore performed with ground-based experiments, where the secondary particles produced by gamma-rays in the atmosphere are imaged and thereby energy and direction of the incident gamma-ray are reconstructed. The effective area is therefore determined by the typical area covered on the ground, resulting in improvements (w.r.t to pair conversion telescopes) by about 4 orders of magnitude at $1 \mathrm{TeV}$.

Imaging the showers is performed either by Cherenkov detectors on ground (water Cherenkov detectors such as MILAGRO) or by Imaging Air Cherenkov Telescopes. Both techniques reach large effective areas on expense of increased energy threshold, which for the former is around a $\mathrm{TeV}$ or the later between 50 and $100 \mathrm{GeV}$. For the signal in question here therefore IACTs are more relevant. Currently, they typically consist of 2 to 5 large size telescopes (mirror diameter $\sim 10-20 \mathrm{~m}$ ), equipped with fast electronic camera recording the very brief and dim bunch of Cherenkov photons produced during the secondary particles shower development in the atmosphere. Several experiments are currently in operation: VERITAS in the United States and MAGIC at La Palma cover the northern hemisphere, whereas H.E.S.S., located in Namibia, is the best suitable experiment to observe the Galactic centre with a low energy threshold. The performance of the 4 telescopes configuration, operational since 2004, is too poor at energies relevant for the potential signal. However, the addition of a much larger 


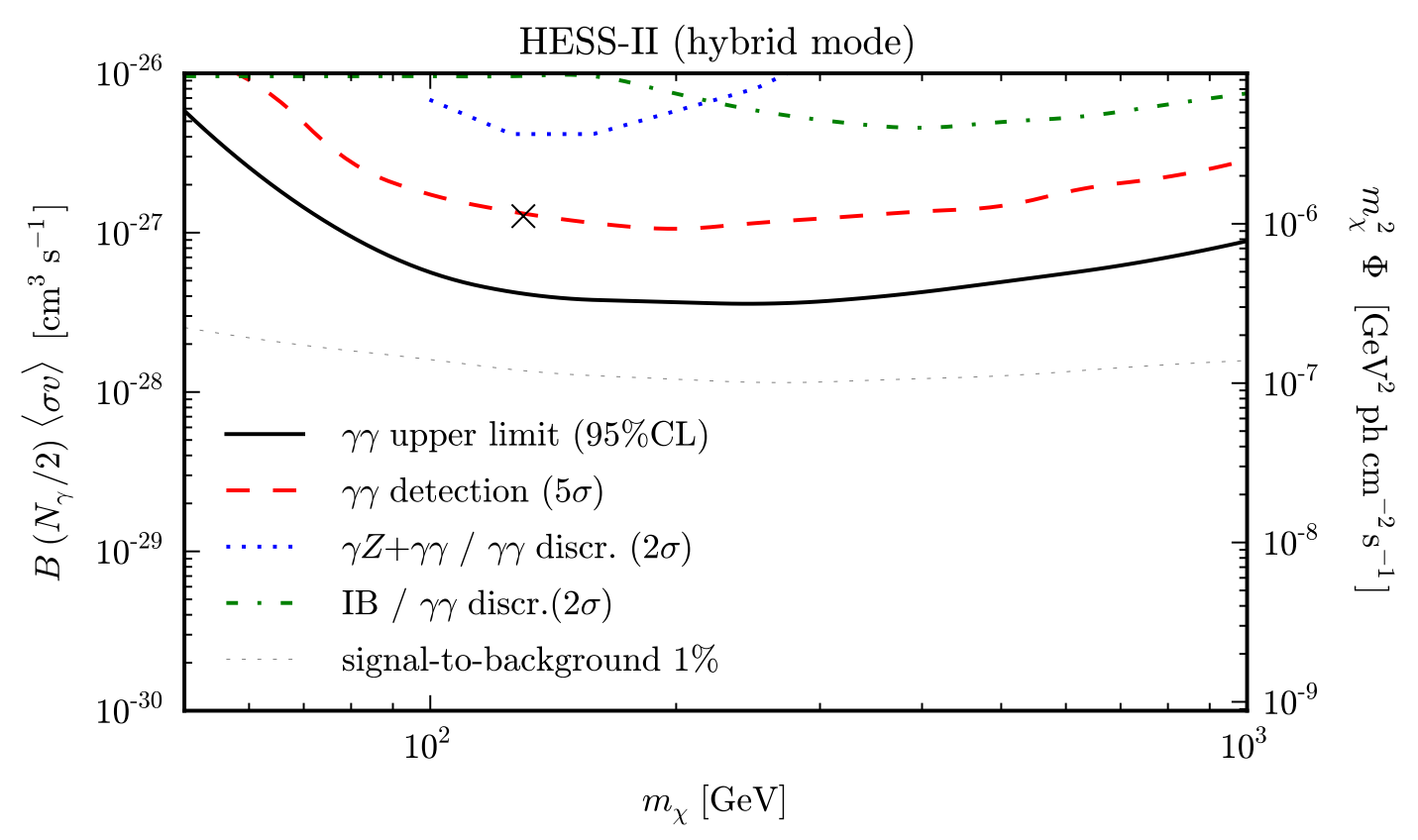

FIG. 5: Same as Fig. 3 but for 50 h of Galactic center observation with HESS-II. The gray dotted line shows cross-sections at which the signal-to-background ratio at the line peak would drop below $1 \%$.

fifth $\left(600 \mathrm{~m}^{2}\right)$ telescope, being currently installed (denoted HESS-II) will allow to record showers initiated by incident particles of lower energy. This is therefore the configuration we focus on. Preliminary estimates of the effective area and energy resolution of HESS-II for an hybrid mode ${ }^{3}$ operation have been reported in [17]. This operating mode will allow to observe events above $50 \mathrm{GeV}$ with an energy resolution of about $20 \%$.

For IACT experiments, several sources of background have to be taken into account. First of all, charged cosmic rays, hitting the Earth with a much greater rate than the most powerful gamma-ray sources, can produce Cherenkov showers mimicking the signal induced by VHE gamma-rays. This is especially true for electron induced showers which displayed very similar properties. Hadronic showers, mainly due to protons, often carry secondary particles with larger transverse momentum with respect to the shower direction, which translate into more scattered images reconstructed in the focal plane of the antennas. Hence only a fraction $\left(\mathcal{O}\left(10^{-2}\right)\right)$ of these events remain.

For the signal region, in case of HESS-II, we adopt a circular target region of $1^{\circ}$ radius around the Galactic centre. As energy window, we adopt two times the window size in Ref. [53]. For observations toward the Galac-

\footnotetext{
3 The hybrid-mode consists of the observation of the same events by the largest telescope and at least one of the $13 \mathrm{~m}$ diameter ones.
}

tic centre, two additional gamma-ray signals have to be taken into account: the detected point-like HESS source HESS J1745-290 [54] and the diffuse emission around the Galactic centre also measured by HESS [55]. For these four components, we used a similar description as the one described in Appendix A of [53].

As for GAMMA-400, we investigated the capabilities of HESS-II to detect a gamma-ray line-like DM signal. We assumed an observation of $50 \mathrm{~h}$ towards the Galactic centre, for intermediate zenith angle $\left(20^{\circ}\right)$. The $95 \%$ C.L. achieved with HESS-II operating in hybrid-mode, should be as low as few $10^{-28} \mathrm{~cm}^{3} \mathrm{~s}^{-1}$ for DM masses between 80 to $1000 \mathrm{GeV}$, see figure 5. Since the energy resolution of HESS-II is almost a factor 10 worse than what should be achieved with GAMMA-400, we clearly see the benefits of a large number of events. However, such energy resolution drastically reduce the possibility to distinguish a two-lines scenario. Nevertheless, like GAMMA-400, HESS-II will be able to confirm the excess reported in [12].

In the near future, Cherenkov Telescope Array (CTA) will be the next generation of imaging atmospheric Cherenkov telescopes. It aims at increasing the sensitivity of the current experiments up to a factor $\sim 10$ in the $\mathrm{TeV}$ domain, and also to lower the energy threshold to few tens of $\mathrm{GeV}$. The current design of the full observatory holds $\geq 60$ telescopes, for which nearly $2 / 3$ will be installed in the southern hemisphere for a better view of the Galactic centre, see [18] for a detailed description of the design and estimates of the performances of the instrument. We use the energy dependence of the energy 


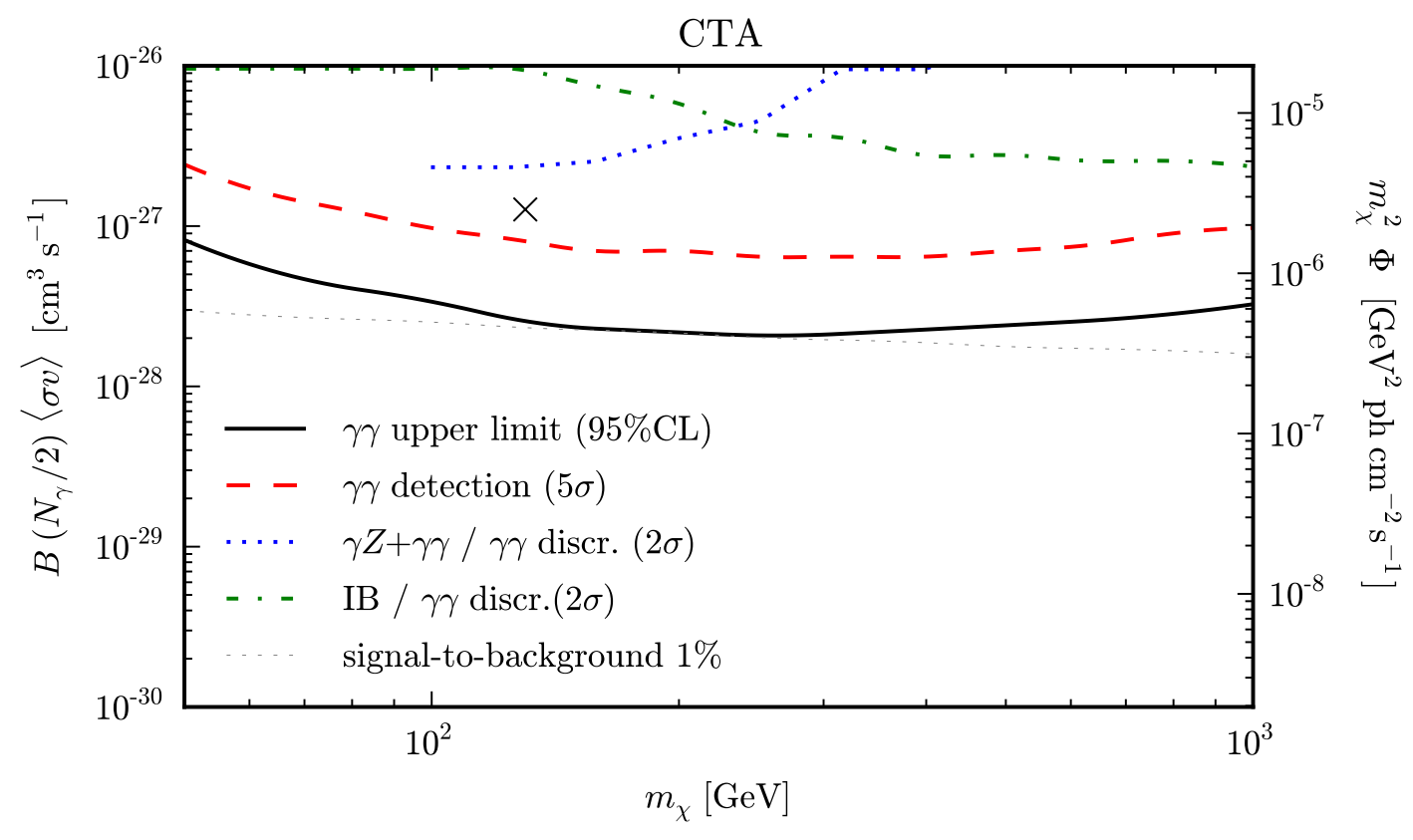

FIG. 6: Same as Fig. 3 but for $5 \mathrm{~h}$ of Galactic center observations with CTA.

resolution of [18], and we also assume the energy dependence of the effective area from [56]. As signal region, we adopt a circular target region of $2^{\circ}$ around the Galactic center.

The signal region definition and background description we adopted for CTA are similar to the ones discussed above for HESS-II. With the presence of several large size telescope in the centre of the array, CTA will be more sensitive than HESS-II. In order to remain in the statistically limited regime, we reduced the observing time for CTA down to 5 hours. At very low energies, since we impose that the showers are observed at least by two telescopes, CTA in its current design will not have a much larger effective area. Moreover the intrinsic fluctuations in the low energy showers limit the energy resolution that will be achieved even with several telescopes, so that in this energy range, we do not expect stronger limits from CTA. Above $80 \mathrm{GeV}$ however, the higher telescopes multiplicity will increase the performance (larger effective area and better energy resolution) so that CTA sensitivity will be almost constant up to $1 \mathrm{TeV}$. From figure [6. we also see that CTA will be very important to probe line-like signals from DM annihilations above $100 \mathrm{GeV}$, since after $5 \mathrm{~h}$ of observations of the Galactic centre, CTA will be more sensitive than five years of GAMMA-400 observations.

\section{DISCUSSION AND CONCLUSIONS}

The detection of a sharp feature at an energy of 130 $\mathrm{GeV}$ in Fermi-LAT data has sparked the interest of the astroparticle community, since the presence of gammaray lines has long been considered a smoking-gun signature of new physics, possibly pointing to the annihilation of dark matter particles. Of course, future Fermi-LAT data will be very important: If the Fermi-LAT collaboration can exclude instrumental effects as the cause of the structure, it may well, in case upcoming data strengthens the feature, confidently establish discovery of the effect. In any case, future gamma-ray observatories would provide necessary independent confirmation and are expected to clarify the experimental situation, in view of their increased effective area or better angular resolution. In particular we focused here on three upcoming experiments: HESS-II, CTA and GAMMA-400.

We summarize here the main results:

- We have calculated the sensitivity to gamma-ray lines for the three experiments, and we have shown that all of them will be able to confirm or rule out the presence of the $130 \mathrm{GeV}$ line. In all cases, in fact, the feature found in Fermi-LAT data would be detectable with a significance higher than $5 \sigma$.

- We have assessed, for each experiment, the prospects for identifying the presence of additional lines, which would allow a better reconstruction of the particle properties of the annihilating dark matter particle. We found that only GAMMA400 , thanks to a claimed energy resolution of about $1.5 \%$ at $100 \mathrm{GeV}$, will be able to separate a $\gamma \gamma$ line from a $Z \gamma$ or $H \gamma$, if the corresponding branching ratio is comparable to that into two photons, while HESS-II and CTA cannot separate them. 
- We investigate for which signal fluxes a signal arising from the annihilation into $\gamma \gamma$ can be distinguished from IB photons, and found that GAMMA400 would be able to distinguish at a $95 \%$ CL between a gamma-ray line and IB photons if the 130 $\mathrm{GeV}$ feature is real, and we have identified the broader region of the parameter space where a discrimination is possible.

HESS-II will soon be operational and given the good performances foreseen for the instrument in hybrid-mode, we stress that it should offer a quick confirmation of the genuineness of the signal reported in [12] (our estimates are based on an exposure time of 50 hours, assuming intermediate zenith angles), since this could provide on a short timescale an independent observation with completely different background and systematic errors. As for CTA, the actual construction of the array should start in 2015 , and the first data should realistically be available by 2018 .

In the case of GAMMA-400, the claimed improvement in energy and angular resolution over Fermi-LAT make it an invaluable tool for dark matter searches. We have demonstrated that it has an enormous potential in the detection and discrimination of lines, despite the smaller effective area compares to the NASA satellite, and we therefore strongly encourage this experimental effort.

\section{Acknowledgments}

We are grateful for discussions with Yvonne Bechereni, Torsten Bringmann, James Buckley, Michael Kuhlen, Emmanuel Moulin, Michael Punch and Hannes-S. Zechlin. The research of LB was carried out under Swedish Research Council (VR) contract no. 621-2009-3915. GB acknowledges the support of the European Research Council through the ERC Starting Grant WIMPs Kairos. CW acknowledges partial support from the European 1231 Union FP7 ITN INVISIBLES (Marie Curie Actions, PITN-GA-2011-289442). JC is research fellow of the Royal Swedish Academy of Sciences financed by a grant of the K\&A Wallenberg foundation.
[1] G. Bertone (ed.), "Particle Dark Matter: Observations, Models and Searches", Cambridge University Press (2010), ISBN:9780521763684

[2] G. Jungman, M. Kamionkowski and K. Griest, Phys. Rept. 267 (1996) 195 hep-ph/9506380; L. Bergstrom, Rept. Prog. Phys. 63 (2000) 793 hep-ph/0002126]; C. Munoz, Int. J. Mod. Phys. A 19 (2004) 3093 hep-ph/0309346; G. Bertone, D. Hooper and J. Silk, Phys. Rept. 405 (2005) 279 hep-ph/0404175.

[3] G. Aad et al. [ATLAS Collaboration], Phys. Lett. B 710 (2012) 49 arXiv:1202.1408 [hep-ex]]; S. Chatrchyan et al. [CMS Collaboration], Phys. Lett. B 710 (2012) 26 arXiv:1202.1488 [hep-ex]].

[4] O. Buchmueller, R. Cavanaugh, A. De Roeck, M. J. Dolan, J. R. Ellis, H. Flacher, S. Heinemeyer and G. Isidori et al., arXiv:1112.3564 [hep-ph]; A. Fowlie, A. Kalinowski, M. Kazana, L. Roszkowski and Y. L. S. Tsai, Phys. Rev. D 85 (2012) 075012 arXiv:1111.6098 [hep-ph]]; S. Profumo, Phys. Rev. D 84 (2011) 015008 arXiv:1105.5162 [hep-ph]]; K. Hagiwara, R. Liao, A. D. Martin, D. Nomura and T. Teubner, J. Phys. G G 38 (2011) 085003 arXiv:1105.3149 [hepph]]; M. Farina, M. Kadastik, D. Pappadopulo, J. Pata, M. Raidal and A. Strumia, Nucl. Phys. B 853 (2011) 607 arXiv:1104.3572 [hep-ph]]; A. Fowlie, M. Kazana, K. Kowalska, S. Munir, L. Roszkowski, E. M. Sessolo, S. Trojanowski and Y. -L. S. Tsai, arXiv:1206.0264 [hepph]; J. Ellis and K. A. Olive, Eur. Phys. J. C 72 (2012) 2005 arXiv:1202.3262 [hep-ph]]; C. Strege, G. Bertone, D. G. Cerdeno, M. Fornasa, R. R. de Austri and R. Trotta, JCAP 1203 (2012) 030 arXiv:1112.4192 [hepph]]; G. Bertone, D. G. Cerdeno, M. Fornasa, R. Ruiz de Austri, C. Strege and R. Trotta, JCAP 1201 (2012) 015 arXiv:1107.1715 [hep-ph]].

[5] G. Bertone, Nature 468, 389 (2010) arXiv:1011.3532 [astro-ph.CO]].
[6] G. Bertone, D. G. Cerdeno, M. Fornasa, R. R. de Austri and R. Trotta, Phys. Rev. D 82 (2010) 055008 arXiv:1005.4280 [hep-ph]].

[7] G. Bertone, D. G. Cerdeno, M. Fornasa, L. Pieri, R. Ruiz de Austri and R. Trotta, Phys. Rev. D 85 (2012) 055014 arXiv:1111.2607 [astro-ph.HE]].

[8] E. A. Baltz, B. Berenji, G. Bertone, L. Bergström, E. Bloom, T. Bringmann, J. Chiang, J. Cohen-Tanugi et al., JCAP 0807 (2008) 013. arXiv:0806.2911 [astro$\mathrm{ph}]$.

[9] M. Ackermann et al. [Fermi-LAT Collaboration], Phys. Rev. Lett. 107 (2011) 241302 arXiv:1108.3546 [astroph.HE]]; A. Geringer-Sameth and S. M. Koushiappas, Phys. Rev. Lett. 107 (2011) 241303 arXiv:1108.2914 [astro-ph.CO]]; A. Geringer-Sameth and S. M. Koushiappas, arXiv:1206.0796 [astro-ph.HE]; I. Cholis and P. Salucci, arXiv:1203.2954 [astro-ph.HE].

[10] M. Ackermann et al., JCAP 1005, 025 (2010) arXiv:1002.2239 [astro-ph.CO]]; X. Huang, G. Vertongen and C. Weniger, JCAP 1201 (2012) 042 arXiv:1110.1529 [hep-ph]]; S. 'i. Ando and D. Nagai, JCAP 1207 (2012) 017 arXiv:1201.0753 [astro-ph.HE]].

[11] T. Bringmann, X. Huang, A. Ibarra, S. Vogl and C. Weniger, arXiv:1203.1312 [hep-ph].

[12] C. Weniger, arXiv:1204.2797 [hep-ph].

[13] E. Tempel, A. Hektor and M. Raidal, arXiv:1205.1045 [hep-ph].

[14] M. Su and D. P. Finkbeiner, arXiv:1206.1616 [astroph.HE].

[15] F. Aharonian, D. Khangulyan and D. Malyshev, arXiv:1207.0458.

[16] Fermi-LAT data server, http://fermi.gsfc.nasa.gov/ssc/data/access/lat/ For a description of the Fermi-LAT instrument, see W. B. Atwood et al. [LAT Collaboration], Astrophys. J. 697 (2009) 1071 arXiv:0902.1089 [astro-ph.IM]]. 
[17] Y. Becherini, A. Djannati-Atai, M. Punch, K. Bernlöhr, S. Ehlert, J. Masbou, E. Moulin, M. Paz Arribas, Proceeding AIPC; J. Lefaucheur, Y. Becherini, S. Pita, M. Punch, Proceeding of Gamma2012, to appear.

[18] CTA Consortium, Exper. Astron. 32 (2011) 193 arXiv:1008.3703 [astro-ph.IM]]; see http://www.cta-observatory.org/

[19] A. M. Galper, O. Adriani, R. L. Aptekar, I. V. Arkhangelskaja, A. I. Arkhangelskiy, M. Boezio, V. Bonvicini and K. A. Boyarchuk et al., arXiv:1201.2490 [astro-ph.IM].

[20] J.F. Navarro, C.S. Frenk and S.D.M. White, Astrophys. J. 462 (1996) 563.

[21] V. Springel, J. Wang, M. Vogelsberger, A. Ludlow, A. Jenkins, A. Helmi, J. F. Navarro and C. S. Frenk et al., Mon. Not. Roy. Astron. Soc. 391 (2008) 1685 arXiv:0809.0898 [astro-ph]].

[22] J. Hisano, S. .Matsumoto, M. M. Nojiri, O. Saito, Phys. Rev. D71 (2005) 063528. hep-ph/0412403; N. ArkaniHamed, D. P. Finkbeiner, T. R. Slatyer and N. Weiner, Phys. Rev. D 79, 015014 (2009) arXiv:0810.0713 [hepph]]; M. Lattanzi and J. I. Silk, arXiv:0812.0360 [astroph]; A. Hryczuk, R. Iengo and P. Ullio, JHEP 1103 (2011) 069 arXiv:1010.2172 [hep-ph]].

[23] S. Galli, F. Iocco, G. Bertone and A. Melchiorri, Phys. Rev. D 80 (2009) 023505 arXiv:0905.0003 [astro-ph.CO]]; T.R. Slatyer, N. Padmanabhan, D.P. Finkbeiner, Phys. Rev. D 80 (2009) 043526, arXiv:0906.1197 G. Huetsi, A. Hektor, M. Raidal, Astron. Astrophys. 505 (2009) 999, arXiv:0906.4550 M. Cirelli, F. Iocco and P. Panci, JCAP 0910 (2009) 009, arXiv:0907.0719 [astro-ph.CO]; D. P. Finkbeiner, S. Galli, T. Lin and T. R. Slatyer, arXiv:1109.6322 [astroph.CO]; T. Kanzaki, M. Kawasaki and K. Nakayama, Prog. Theor. Phys. 123 (2010) 853, arXiv:0907.3985 [astro-ph.CO]; Q. Yuan, B. Yue, X.-J. Bi, X. Chen, X. Zhang, JCAP 1010 (2010) 023, arXiv:0912.2504 G. Hutsi, J. Chluba, A. Hektor and M. Raidal, Astron. Astrophys. 535 (2011) A26 arXiv:1103.2766 [astroph.CO]]. S. Galli, F. Iocco, G. Bertone and A. Melchiorri, Phys. Rev. D 84 (2011) 027302 arXiv:1106.1528 [astroph.CO]]. A. Natarajan, Phys. Rev. D 85 (2012) 083517 arXiv:1201.3939 [astro-ph.CO]].

[24] V. Springel, S. D. M. White, C. S. Frenk, J. F. Navarro, A. Jenkins, M. Vogelsberger, J. Wang, A. Ludlow et al., Nature 456N7218 (2008) 73-80.

[25] L. Pieri, J. Lavalle, G. Bertone and E. Branchini, Phys. Rev. D 83 (2011) 023518 arXiv:0908.0195 [astroph.HE]].

[26] G. Bertone and D. Merritt, Phys. Rev. D 72 (2005) 103502 astro-ph/0501555; G. Bertone and D. Merritt, Mod. Phys. Lett. A 20 (2005) 1021 astro-ph/0504422; D. Merritt, S. Harfst and G. Bertone, Phys. Rev. D 75 (2007) 043517 astro-ph/0610425; P. Gondolo and J. Silk, Phys. Rev. Lett. 83 (1999) 1719 astro-ph/9906391.

[27] T. E. Jeltema, J. Kehayias, S. Profumo, Phys. Rev. D80 (2009) 023005. arXiv:0812.0597] [astroph]]; M. A. Sanchez-Conde, M. Cannoni, F. Zandanel, M. E. Gomez, F. Prada, arXiv:1104.3530 [astro-ph.HE]]; A. Pinzke, C. Pfrommer, L. Bergström, arXiv:1105.3240 [astro-ph.HE]]; L. Gao, C. S. Frenk, A. Jenkins, V. Springel, S. D. M. White, arXiv:1107.1916 [astroph.CO]].
[28] L. Bergström and J. Kaplan, Astropart. Phys. 2 (1994) 261 hep-ph/9403239; P. Ullio and L. Bergström, Phys. Rev. D 57 (1998) 1962 hep-ph/9707333; A. Rajaraman, T. M. P. Tait and D. Whiteson, arXiv:1205.4723 [hep-ph].

[29] L. Bergström and H. Snellman, Phys. Rev. D 37 (1988) 3737. L. Bergström, P. Ullio and J. H. Buckley, Astropart. Phys. 9, 137 (1998) arXiv:astro-ph/9712318; G. Bertone, C. B. Jackson, G. Shaughnessy, T. M. P. Tait and A. Vallinotto, JCAP 1203 (2012) 020 arXiv:1009.5107 [astro-ph.HE]]; G. Bertone, C. B. Jackson, G. Shaughnessy, T. M. P. Tait and A. Vallinotto, Phys. Rev. D 80 (2009) 023512 arXiv:0904.1442 [astroph.HE]]; C. B. Jackson, G. Servant, G. Shaughnessy, T. M. P. Tait and M. Taoso, JCAP 1004 (2010) 004 arXiv:0912.0004 [hep-ph]].

[30] T. Bringmann, X. Huang, A. Ibarra, S. Vogl and C. Weniger, arXiv:1203.1312 [hep-ph].

[31] L. Bergström, arXiv:1205.4882 [astro-ph.HE].

[32] L. Bergström, Phys. Lett. B225 (1989) 372.

[33] T. Bringmann, L. Bergström and J. Edsjö, JHEP 0801 (2008) 049 arXiv:0710.3169 [hep-ph]].

[34] A. Birkedal, K. T. Matchev, M. Perelstein and A. Spray, arXiv:hep-ph/0507194

[35] H. Goldberg, Phys. Rev. Lett. 50 (1983) 1419.

[36] E. A. Baltz and L. Bergström, Phys. Rev. D 67 (2003) 043516 arXiv:hep-ph/0211325.

[37] P. Gondolo, J. Edsjö, P. Ullio, L. Bergström, M. Schelke and E. A. Baltz, JCAP 0407, 008 (2004) arXiv:astro-ph/0406204; P. Gondolo, J. Edsjö, P. Ullio, L. Bergström, M. Schelke, E.A. Baltz, T. Bringmann and G. Duda, http://www.physto.se/ edsjo/darksusy.

[38] A. A. Abdo et al. [Fermi-LAT Collaboration], JCAP 1004, 014 (2010) arXiv:1002.4415 [astro-ph.CO]].

[39] A. A. Abdo et al., Phys. Rev. Lett. 104, 091302 (2010) arXiv:1001.4836 [astro-ph.HE]].

[40] M. Ackermann et al. [LAT Collaboration], arXiv:1205.2739 [astro-ph.HE].

[41] G. Vertongen and C. Weniger, JCAP 1105 (2011) 027 arXiv:1101.2610 [hep-ph]].

[42] A. R. Pullen, R. -R. Chary and M. Kamionkowski, Phys. Rev. D 76 (2007) 063006 [Erratum-ibid. D 83 (2011) 029904] astro-ph/0610295.

[43] A. Ibarra, S. Lopez Gehler and M. Pato, arXiv:1205.0007 [hep-ph]; E. Dudas, Y. Mambrini, S. Pokorski and A. Romagnoni, arXiv:1205.1520 [hep-ph]; J. M. Cline, arXiv:1205.2688 [hep-ph]; K. -Y. Choi and O. Seto, arXiv:1205.3276 [hep-ph]; B. Kyae and J. -C. Park, arXiv:1205.4151 [hep-ph]; H. M. Lee, M. Park and W. -I. Park, arXiv:1205.4675 [hep-ph]; A. Boyarsky, D. Malyshev and O. Ruchayskiy, arXiv:1205.4700 [astroph.HE]; B. S. Acharya, G. Kane, P. Kumar, R. Lu and B. Zheng, arXiv:1205.5789 [hep-ph]; M. R. Buckley and D. Hooper, arXiv:1205.6811 [hep-ph]; A. GeringerSameth and S. M. Koushiappas, arXiv:1206.0796 [astroph.HE]; X. Chu, T. Hambye, T. Scarna and M. H. G. Tytgat, arXiv:1206.2279 [hep-ph]; D. Das, U. Ellwanger and P. Mitropoulos, arXiv:1206.2639 [hep-ph]; N. Weiner and I. Yavin, arXiv:1206.2910 [hep-ph].

[44] S. Profumo and T. Linden, arXiv:1204.6047 [astroph.HE].

[45] G. Dobler, D. P. Finkbeiner, I. Cholis, T. R. Slatyer and N. Weiner, Astrophys. J. $\mathbf{7 1 7}$ (2010) 825 arXiv:0910.4583 [astro-ph.HE]]; M. Su, T. R. Slatyer and D. P. Finkbeiner, Astrophys. J. 724 (2010) 1044 
arXiv:1005.5480 [astro-ph.HE]].

[46] M. Kuhlen, private communication.

[47] S.S. Wilks, The Annals of Mathematical Statistics 9 (1938) 60-62;

[48] Houck, J. C., Denicola, L. A., Astronomical Data Analysis Software and Systems IX, 216 (2000) 591.

[49] W. A. Rolke, A. M. Lopez and J. Conrad, Nucl. Instrum. Meth. A 551 (2005) 493 physics/0403059.

[50] H. Chernoff, Ann. Math. Statist. 25 (1954) 573-578.

[51] F. James, Statistical methods in experimental physics, Hackensack, USA, World Scientific (2006) 345 p.
[52] J. Conrad, H. Dickinson, in preparation.

[53] T. Bringmann, F. Calore, G. Vertongen and C. Weniger, Phys. Rev. D 84 (2011) 103525 arXiv:1106.1874.

[54] F. Aharonian et al. [HESS Collaboration], Phys. Rev. Lett. 97 (2006) 21102 [Erratum-ibid. 97 (2006) 249901] arXiv:astro-ph/0610509.

[55] F. Aharonian et al. [HESS Collaboration], Nature 439 (2006) 695 [arXiv:astro-ph/060321].

[56] Contribution from the CTA Consortium, Proceedings of the 31st ICRC, arXiv:1111.2183. 